\title{
Kernos
}

Revue internationale et pluridisciplinaire de religion grecque antique

26 | 2013

Varia

\section{Oracoli e teologia: praxis oracolare e riflessioni}

\section{Giulia Sfameni Gasparro}

\section{(2) OpenEdition \\ Journals}

\section{Edizione digitale}

URL: http://journals.openedition.org/kernos/2208

DOI: $10.4000 /$ kernos. 2208

ISSN: 2034-7871

\section{Editore}

Centre international d'étude de la religion grecque antique

\section{Edizione cartacea}

Data di pubblicazione: 10 octobre 2013

Paginazione: 139-156

ISSN: 0776-3824

\section{Notizia bibliografica digitale}

Giulia Sfameni Gasparro, « Oracoli e teologia: praxis oracolare e riflessioni », Kernos [En ligne], 26 |

2013, mis en ligne le 31 octobre 2015, consulté le 02 mars 2021. URL : http://

journals.openedition.org/kernos/2208; DOI : https://doi.org/10.4000/kernos.2208 


\title{
Oracoli e teologia: praxis oracolare e riflessioni teologiche nella tarda antichità
}

\begin{abstract}
Résumé : L'« oracle théologique », phénomène typique de l'Antiquité tardive, est une mutation du genre traditionnel du chresmós, parole révélée par une divinité à une communauté civique ou à un individu, habituellement dans une institution publique, avec un sanctuaire et du personnel sacerdotal. Des centres apolliniens comme Didymes et Claros, et de nouvelles institutions comme celle du «nouvel Asclépios », Glycon, énoncent des oracles fournissant des réponses aux interrogations sur la nature du divin et sur la destinée de l'homme après la mort. Dans ces réponses se dessine une nouvelle «théologie », qui envisage une structure pyramidale et graduée, avec un sommet principal, transcendant, d'une part, et, d'autre part, caractérisé par des traits cosmiques, auquel sont subordonnées de nombreuses puissances, actives dans le monde, identifiées aux dieux traditionnels.
\end{abstract}

Abstract: The "theological oracle", a phenomenon typical of the late Antiquity, represents a mutation of the traditional genre of the chresmós, a word revealed by a deity to a city community or to a single individual, usually within a public institution, with a sanctuary and religious functionaries. Apollonian temples, such as those at Didyma and Claros, and new institutions such as the "neos Asklepios", Glykon of Abonuteichos, provide oracles to give answers to questions on the nature of the divine and on the destiny of man after death. In these responses there also emerges a new "theology", with a graduated pyramidal structure, displaying a supreme principle, transcendent on the one hand and with cosmic traits on the other, to which many powers are subordinated, active in the world, identified with the traditional gods.

\section{Introduzione:}

\section{tempi e modalità del genere oracolare "teologico"}

Una singolare definizione dell'Apollo delfico quale «filosofo non meno che indovino» ${ }^{1}$, è proposta da Plutarco che la giustifica ritenendo che il dio "porti rimedio alle difficoltà della vita e le risolva dando i suoi responsi a chi lo consulta; invece, le difficoltà che riguardano la ragione, è lui che le fa sorgere e le presenta a chi è filosofo per natura, istillando nell'anima un desiderio che conduce alla

${ }^{1}$ De E delph., 2, 385b; ed. e trad. Moreschini (1997), p. 68-69. Cf. 6, 386e in cui il dio delfico è definito «dialettico al massimo grado». Si veda l'edizione dei Dialoghi pitici di FLACELIÈrE (1974). Dopo una momentanea eclissi, l'oracolo delfico -come altrove nota lo stesso Plutarcoconosce in questo periodo una rinnovata popolarità. Su Delfi e gli altri centri oracolari apollinei e in genere sul fenomeno divinatorio in Grecia basti segnalare qui la recente sintesi di JOHNSTON (2008) con la bibliografia essenziale. 
veritàı ${ }^{2}$. Tale definizione può esser posta in epigrafe al fenomeno di cui intendo brevemente discutere. Infatti, da quando A.D. Nock, in un saggio del 19283 , ha usato la formula "oracles théologiques" per designare un'ampia produzione oracolare che -a partire dal II sec. d.C.- si manifesta con grande frequenza in documenti diversi, epigrafici e letterari, esprimendo forti interessi religiosi, di tipo specificamente "teologico" (nel senso originario del termine in ambito greco, quale "discorso sul divino") 4 , essa è divenuta corrente nel linguaggio scientifico per circoscrivere un fenomeno peculiare dell'orizzonte culturale del mondo mediterraneo in età tardo-antica. Esso infatti rivela una particolare mutazione del genere tradizionale greco dell'»oracolo» (chresmós), quale parola rivelata da una divinità, solitamente in rapporto ad un'istituzione cittadina, con santuario e personale sacro, ad una comunità o a singoli individui, in seguito ad una consultazione relativa, di norma, a situazioni di crisi per l'una o per gli altri, con indicazione del comportamento utile al conseguimento dei risultati positivi sperati.

Con un'espressione egualmente icastica usata da P. Batiffol che di fatto aveva inaugurato la ricerca sul tema in un contributo del 1916, al seguito della fondamentale monografia di K. Buresch sull'oracolo di Claros, si può parlare di “oracles professeurs de théologie", relativi all'identità dell'una o dell'altra divinità del pantheon tradizionale ovvero talora, con forte tensione religiosa, a dare soluzione al più vasto e drammatico quesito: "Chi è dio?". In questa produzione oracolare si delinea anche una nuova "teologia", che contempla una struttura piramidale e graduata, con un sommo principio, trascendente per un verso e dai tratti cosmici per l'altro, a cui sono subordinate numerose potenze, attive nel mondo, identificate con gli dei tradizionali ${ }^{6}$. Si impone pertanto il problema di indagare le ragioni di tale "mutazione" genetica e di circoscrivere ambienti e circostanze storiche di un evento che talora appare connesso a centri cultuali tradizionali, come appunto il grande santuario apollineo di Claros, presso Colofone, e quello di Didima, presso Mileto, ma pure si manifesta in maniera "epidemica" nei più diversi contesti, coinvolge autori anche di alto spessore filosofico come Porfirio, interessati a raccogliere e commentare oracoli attribuiti proprio all'uno o all'altro di quei centri ovvero di provenienza non dichiarata, e trova voce nel quadro di singolari creazioni religiose, come quella del «(falso) profeta» di Abonuteico, Alessandro, oggetto della critica feroce di Luciano. Né il fenomeno conosce barriere di

${ }^{2}$ De E delph., 1, 384e-f. Cf. ibid., 6, 386e-f: «allo stesso modo, dunque, il dio, quando manifesta dei responsi ambigui, rafforza e organizza l'arte dialettica, in quanto essa è necessaria per coloro che in futuro vorranno intenderlo rettamente». Ed. e trad. MORESCHINI (1997), p. 7879. Cf. SFAMENi Gasparro (1996).

${ }^{3}$ Nock (1928).

${ }^{4}$ Sul tema cf. Sfameni Gasparro (1994).

${ }^{5}$ Batiffol (1916), p. 193. Cf. Buresch (1889).

${ }^{6}$ Una discussione di questa problematica in SFAMENI GASPARro (2010). 
appartenenza etnica o religiosa, coinvolgendo esso -nel grande amalgama socioculturale e religioso dei primi secoli dell'Impero- i tre grandi versanti che lo compongono, ossia quello delle culture tradizionali a impianto politeistico e forte densità ellenica o ellenizzata, quello della tradizione giudaica nella forma peculiare della rivelazione sibillina ${ }^{7}$, con la quale questa tradizione travalica i rigorosi confini del proprio geloso isolamento religioso e si apre a un programmatico "meticciamento" con il versante "pagano" in cui tale tradizione ha avuto origine e infine il versante cristiano. A quest'ultimo, di fatto, dobbiamo memoria della più ampia parte degli Oracula hellenica, sia negli autori che li usano in funzione polemica e apologetica, come un Eusebio o un Lattanzio", sia nella confezione di "raccolte" in qualche misura autonome, anche se ispirate dagli stessi intenti, come nel caso emblematico dell'anonimo bizantino (V-VI sec.), autore della Theosophia contenente gran numero di «Oracoli degli dèi ellenici», notoriamente referente fondamentale di tutte le ricerche sul tema9. Senza poter esplorare in questa sede un così vasto scenario mi limiterò a sottoporre ad una nuova verifica critica la documentazione relativa ad un episodio che -all'interno di esso- propone il tema della convergenza di una struttura istituzionale a forte densità iatromantica solidamente costruita e di istanze di tipo "teologico". Tale "episodio" è tanto più significativo in quanto costituisce una fondazione religiosa nuova che, pur modellata su schemi tradizionali, coagula una serie di temi e di tendenze religiose tipici del proprio tempo, quel II sec. d.C. che vede appunto, contestualmente a nuove forme di religiosità e di concezioni dei rapporti uomo-divinità, la mutazione oracolare in direzione della ricerca di risposte alle aporie del logos.

\section{L'oracolo di Glicone: un falso storico o una fonte di "oracoli teologici"?}

Si tratta di quella singolare creazione religiosa costituita dall'oracolo di Glicone, fondato nella città di Abonutico in Paflagonia da un Alessandro ${ }^{10}$ che Luciano definisce «falso profeta» ${ }^{11}$, la cui vicenda umana si conclude intorno al

\footnotetext{
7 Inutile in questa sede, oltre che impossibile, sarebbe ogni tentativo di fornire un sia pur minimo bilancio storiografico sul tema, peraltro di fondamentale impatto nello scenario religioso tardo-antico (cf. SFAMENI GASPARRo 1998a). Segnalo soltanto la nuova edizione della traduzione degli Oracoli giudeo-cristiani di SUÁREZ DE LA TORRE (2002²), con aggiornamento bibliografico, per il quale si veda anche MONACA (2008).

${ }^{8}$ Analisi della tradizione patristica che appella agli oracoli pagani, sia in funzione polemica sia in funzione apologetica, quali testimonia a sostegno della stessa rivelazione biblica, dopo BUSINE (2005), p. 318-431, in NiETO IBÁÑEZ (2011).

${ }^{9}$ BEATRICE (2001).

10 Per una più ampia e articolata analisi della facies religiosa del personaggio e della sua fondazione religiosa $\mathrm{mi}$ sia permesso rimandare a miei precedenti contributi con relativa documentazione: SFAMENI GASPARRO (1996 [1998]) e (1999).

11 Tra le edizioni e traduzioni del testo, si segnala in ultimo VICTOR (1997) e CASTER-DANZAT $\left(2002^{2}\right)$. Si veda anche la traduzione di Campolunghi in Del Corno-Campolunghi (1992), da cui
} 
170 d.C. Definito come un bios del «ciarlatano» (goes) redatto in forma di biblion su richiesta di un amico, Celso, e a lui inviato per smascherare le sue «invenzioni (epinoiai), atti audaci (tolmemata) e trucchi magici (magganeiai)» (Alex., 1), il testo di Luciano delinea -con tutte le risorse di un'abilità retorica che non ha bisogno di dimostrazioni- un quadro vivido e mosso della personalità e delle azioni del personaggio. Secondo le regole del genere biografico egli traccia innanzitutto le linee fondamentali della prima, consegnandoci l'immagine di un vero e proprio theios aner: bello nell'aspetto, «simile a un dio (theoprepes)», di intelligenza superiore ad ogni altro e dalle doti eccezionali, peraltro subito connotate in luce negativa in quanto rivolte all'inganno e alla mistificazione, poiché -per la sua straordinaria capacità di persuasione e sotto l'apparenza della virtù- egli era capace di compiere le azioni più abominevoli. Segue quindi la descrizione di queste ultime, dall'educazione presso un «ciarlatano» (goes) esperto nelle arti magiche e nella medicina, a sua volta discepolo di Apollonio di Tiana (Alex., 5) ${ }^{12}$, all'associazione con un «compositore di inni (chorographos)», Cocconas di Bisanzio, e con una ricca dama di Pella. Presso la residenza di costei scatta la molla che darà inizio a tutta l'avventura: l'esperienza della tipica fauna locale, costituita da serpenti grandi e mansueti, fa nascere nei due amici il progetto della creazione di un nuovo manteion in cui questo animale, dalle note connessioni con il culto iatromantico di Asclepio, giocherà un ruolo centrale come manifestazione e «impersonazione» di un «nuovo Asclepio», denominato Glicone, «il dolce». I momenti forti della vicenda sono la scelta di Calcedonia, sede di un famoso manteion di Apollo ${ }^{13}$ dove vengono nascoste alcune tavolette bronzee con oracoli preannuncianti l'imminente epifania di Asclepio e di Apollo ad Abonutico (Alex., 10), l'inizio della costruzione di una sede sacra in questa cittadina e la manifestazione carismatica di Alessandro che, a sua volta annunciato da oracoli «di riconoscimento» ${ }^{14}$ che proclamano la sua qualità profetica e l'ascendenza eroica e divina ${ }^{15}$, si manifesta nelle forme tipiche dell'invasamento religioso ed estrae dall'acqua della fonte posta presso il tempio un uovo da cui emerge, nella forma di un piccolo serpente, il nuovo Asclepio (Alex., 9-14). Quindi, all'interno di un oikiskos, nella penombra, il profeta si espone alla vista della folla ormai accorsa nella città all'annunzio dell'evento, abbigliato in maniera sontuosa e avvolto dalle spire del grande serpente di Pella, la cui testa, nascosta accuratamente da Alessandro, è stata

cito. Sulla complessa facies ideologica e culturale dell'autore esiste una bibliografia amplissima, notevolmente aumentata negli ultimi decenni in cui si moltiplicano gli studi sull'arte retorica e la Seconda Sofistica che a vario titolo lo chiamano in causa. Utili rimangono ancora i contributi di CASTER (1937); BOMPAIRE (1958); SCHWARTZ (1965); JONES (1986) e BRANHAM (1989).

12 Sull'importanza di questa testimonianza si veda ROBIANO (2001).

${ }^{13}$ In ultimo cf. RoBU (2007).

${ }^{14} \mathrm{Si}$ veda la tipologia oracolare proposta da $\operatorname{AunE}\left(1983,1991^{2}, 1996\right)$, p. 137.

15 Alex., 11: «Perseide di stirpe, caro a Febo, ecco alla vostra presenza / il divino Alessandro, che per volere del fato ha il sangue di Podalirio». L'oracolo della Sibilla annuncia la nascita di un profeta «sulle rive del Ponto Eussino, presso Sinope». 
sostituita da una fittizia a forma umana. Da questo momento ha inizio l'attività del manteion, organizzata con un numeroso e vario personale sacro (Alex., 16: keryx e theologos; 23-24), ma interamente fondata sulla persona del profeta, a cui appellano le diverse forme di consultazione che assommano le tre principali tipologie oracolari dell'epoca: la redazione di domande e risposte scritte, l'interrogazione diretta -qui configurata nella forma dell'oracolo autofono con l'uso di tecniche note da altre fonti ${ }^{16}$ - e l'incubazione, di cui peraltro è protagonista Alessandro piuttosto che il singolo fedele. Oggetto delle consultazioni sono insieme i problemi delle comunità (epidemie, guerre, carestie, terremoti: Alex., 36) e dei singoli individui, siano essi importanti protagonisti della vita sociale, politica e militare, tra cui emerge il senatore Rutiliano divenuto genero dello stesso Alessandro e addirittura lo stesso Imperatore Marco Aurelio che avrebbe accettato un oracolo di Glicone relativo alla guerra con i Marcomanni e i Quadi (Alex., 48), siano comuni individui preoccupati del proprio avvenire e alla ricerca di rimedi per le malattie. Al favore imperiale si attribuisce un evento di eccezionale portata politica, ossia il mutamento del nome della città in Ionopolis e la coniazione di un tipo monetale con l'immagine di Glicone su un verso e quella del suo profeta sull'altro (Alex., 58). Infine, alcuni singolari oracoli rispondono a interrogativi sull'identità dello stesso titolare dell'oracolo, sulla veridicità degli oracoli apollinei e sul destino ultraterreno dell'anima, inserendosi a loro modo nella tipologia degli «oracoli teologici», di cui pertanto costituirebbero una delle più antiche manifestazioni. Un'ulteriore componente della nuova fondazione religiosa, infine, è stata - a dire del «biografo» - la creazione di una prassi misterica, secondo le più tipiche strutture del genere e in particolare secondo il modello eleusino.

E' nota la consolidata tradizione storiografica che riconosce all'opera lucianea una sostanziale veridicità ${ }^{17}$, sostenuta del resto da numerose testimonianze storiche sui personaggi menzionati e sugli eventi narrati dal «biografo», su cui non è possibile ora diffondersi in dettaglio ${ }^{18}$. Basti soltanto ricordare alcuni dati incontrovertibili: se nessuna fonte oltre Luciano ricorda il profeta-fondatore

\footnotetext{
16 Sulla tecnica di inserire trachee di gru nella testa fittizia di Glicone per dare responsi si veda la testimonianza di Ippolito relativa alle più comuni pratiche magiche del tempo (Ref. IV, 28, 9). Cf. Ref. IV, 34, 1-4 sulle tecniche magiche di apertura e ripristino dei sigilli apposti alle domande oracolari, corrispondenti in larga misura a quelle descritte da Luciano come utilizzate da Alessandro (Alex., 20-21).

17 Un'analisi del trattato in CASTER (1938). Sulla singolare esperienza del profeta di Paflagonia si vedranno ancora utilmente CUMONT (1887); NOCK (1928a); LANE FOX (1986, 1991), p. 254-264. La grande popolarità e la diffusione del culto del serpente Glicone, confermate da reperti archeologici e fonti numismatiche, sono illustrate con grande ricchezza di dati e pertinenza di argomentazioni da ROBERT (1980), p. 393-421. Cf. ancora Robert (1981); AMELING (1985); BordenAsCHE BATTAGLiA (1988); Miron (1996), con puntuale analisi dell'iconografia di Glicone. La significativa documentazione numismatica già in BABELON (1900). Dopo i miei interventi, segnalo anche MASTROCINQUE (1999) e (2010) e CHANIOTIS (2002) che parimenti riconoscono un sostanziale valore storico alla testimonianza lucianea, al di là delle ovvie amplificazioni retoriche e polemiche.

${ }^{18}$ Essi sono citati e discussi nei saggi menzionati, e in particolare in quelli di L. Robert.
} 
dell'oracolo, la mutazione del nome della città -evento di portata sociale e politica eccezionale- è attestata dalla documentazione numismatica, che pure reca la figura di Glicone in numerosi esemplari, anche di altre città (sebbene non vi appaia quella di Alessandro) confermando la contestualità dei due eventi. Né è necessario insistere sul peculiare carattere identitario della iconografia della monetazione dei centri cittadini, anche nello scenario "globalizzato" dell'Impero' ${ }^{19}$. L'iconografia del nuovo Asclepio è attestata inoltre dalla famosa statua rinvenuta a Tomi, da statuette (-amuleto?) ritrovate ad Atene e da una singolare gemma in cui, all'immagine del Decano Chnoubis dalle peculiari facoltà medicali e dall'analoga forma serpentina si accompagna il nome di lui, quello di Glicone e quello tipico della sfera magica, che lo definisce «universalmente valido», di $\mathrm{IaO}^{20}$. Alcuni recenti interventi, tuttavia, hanno messo in dubbio non solo singoli episodi ovvero i particolari della descrizione lucianea, ma la storicità stessa dell'oracolo ${ }^{21}$, ritenuto frutto di un' "invenzione letteraria" di Luciano. Senza potere entrare nel merito del problema con dettagliata discussione dei suoi diversi aspetti, dico soltanto che tale interpretazione, non sostenibile sulla base della nostra documentazione, pare frutto di una diffusa tendenza "decostruzionista" nei nostri studi, spesso alimentata dalla presunzione di noi moderni, scaltriti interpreti del passato e pronti ad applicare ad esso i modelli esegetici più alla moda, ma dimentichi spesso che i protagonisti di esso sapevano bene ciò di cui trattavano. Soprattutto essi, nel redigere le proprie opere, si rivolgevano ai contemporanei che ne potevano verificare le affermazioni, quando fossero relative a eventi di cui tutti erano partecipi. Tale di fatto è la situazione di Luciano e del suo interlocutore, l"'epicureo" Celso ${ }^{22}$, sicchè -pur facendo le inevitabili

\footnotetext{
${ }^{19}$ Basti rimandare ai saggi editi da HowgEgo - HeUCHERT - BurnetT (2005).

${ }^{20}$ MASTROCinQue (1999), p. 146-148.

${ }^{21}$ Sulla base di recenti ricerche relative allo stile fortemente "immaginifico" di Luciano e alla carica polemica della sua opera, POZZI (2003) insiste sul valore sostanzialmente retorico del trattato su Alessandro, mentre BENDLIN (2006, 2011) ne contesta radicalmente la "veridicità" storica, con numerose puntate ironiche sulla "ingenuità" degli studiosi che gli hanno dato credito. Piuttosto "ingenua" tuttavia mi pare piuttosto la notazione dello studioso, secondo cui l'assenza di resti archeologici del manteion di Glicone sarebbe prova della sua inconsistenza storica. Su questa linea esegetica, anche se meno radicale, la posizione di ELM VAN DER OSTEN (2006), che vede sostanzialmente una "costruzione letteraria" nel ruolo di fondatore attribuito ad Alessandro e interpreta lo stesso io narrante/Luciano quale costrutto retorico, non sovrapponibile alla personalità di Luciano/autore. Anche le recenti osservazioni di VAN NUFFELEN (2011), sull'attività letteraria di Luciano (p. 179-199) privilegiano la "strategia della satira" e l'uso di modelli retorici sui referenti "storici" del suo discorso. Lo studioso interpreta l'Alessandro in una singolare prospettiva antiepicurea, oltre che come strumento di polemica contro i culti misterici. Sembra di poter concludere da questa rapida rassegna di opinioni che il testo lucianeo possa servire a dimostrare tutto e il contrario di tutto, secondo i presupposti teorici di ciascun interprete.
}

${ }^{22}$ Non affronto la vexata quaestio dell'identità del personaggio, non essendo possibile dirimere con sicurezza in senso positivo o negativo il problema della sua identificazione con il Celso autore dell'Alethès Logos confutato da Origene. A mio parere, comunque, a tale conclusione fa ostacolo la facies sostanzialmente platonica dell'autore noto all'Alessandrino e soprattutto la fiducia da lui espressa nella validità e veridicità delle numerose "voci" oracolari del panorama 
parti alla fervida capacità inventiva dell'Autore- non si potrà negare che egli intenda descrivere un fenomeno reale, di cui il «narratore»-che alla fine del racconto si identifica con l'autore / Luciano- dichiara di essere stato testimone, citando uomini ed eventi storici altrimenti verificabili. La disinvolta operazione "decostruttiva" del testo lucianeo come riflesso di una vicenda storica, sia pure più o meno profondamente "rivisitata" in chiave polemica e denigratoria e con indubbi interventi di "invenzione" (tutti peraltro modulati su schemi consolidati e ben noti nell'ambiente contemporaneo, tali da potere dunque essere integrati nell'immagine del fenomeno descritto ${ }^{23}$, è portata a termine nell'opera recente di Petsalis-Diomidis ${ }^{24}$ che ha come oggetto un altro fervente devoto di un dio oracolare e medico, Asclepio, ossia il famoso e dotto retore Elio Aristide. La studiosa, che pure analizza in dettaglio la documentazione, soprattutto monumentale, epigrafica e numismatica - prova incontrovertibile del carattere pubblico del culto di Glicone e della sua lunga vitalità tra II e III sec. d.C.- riduce tale culto a una semplice "variante locale" di quello di Asclepio.

Nessuno certo può mettere in dubbio la connessione strutturale fra i due fenomeni ma appare indizio di scarsa sensibilità storica trascurare la forte carica innovativa e creativa inerente al nuovo culto oracolare e medicale, in quanto legato a una figura profetica che agglutina attorno a sé tutti i poteri del proprio dio, fino ad operare una sorta di simbiosi, anche fisica, con esso ${ }^{25}$. La studiosa, tuttavia, se non può negare l'esistenza storica del culto di Glicone, sia pure come un Asclepio "mascherato" in forma serpentina, ritiene di poter concludere che Alessandro, "il falso profeta" non è altro che un'invenzione di Luciano, il cui discorso, in questo come in altri trattati, sarebbe ispirato dall'intento di sottolineare e rafforzare il tema sofistico della dicotomia popolo/élite, credulità/fanatismo e cultura/irrazionalità. Ci si chiede allora se il Celso

religioso tradizionale, contrapposte alla tradizione profetica giudaica e cristiana (cf. SFAMENI GASPARro [1995]). Non c'è bisogno di sottolineare infatti come il postulato fondamentale della posizione epicurea - di cui il Celso lucianeo è detto sostenitore- fosse al contrario la nozione dell'assoluto distacco degli dei dalle faccende cosmiche e umane, con il conseguente rifiuto della divinazione, pur nell'ammissione della loro esistenza.

23 Sulla tipica impostazione parodistica della creazione letteraria lucianea cf. BOMPAIRE (1958), passim e in particolare p. 605-621 dove, a proposito del nostro testo e di quello molto simile del Peregrinus, conclude: «En vérité, le Peregrinus et l'Alexandre sont des sommes parodiques, comme ils sont des sommes de procédés polémiques» (p. 618). Lo studioso tuttavia non nega il "nocciolo" storico delle vicende narrate.

${ }^{24}$ La studiosa non cita in bibliografia né il lavoro di BENDLIN, pubblicato originariamente nel 2006, né quelli di POZZi (2003) e di Elm VAN DER OSTEN (2006), che concordano con la sua argomentazione.

25 Si pensi alla prima manifestazione di Glicone, le cui spire serpentine avvolgevano il corpo del profeta (Alex., 15-16). La pratica degli «oracoli autofoni», che il dio comunicava attraverso Alessandro, e la circostanza che dopo la morte di questo nessuno fu autorizzato a sostituirlo, rafforzano la nozione della stretta simbiosi fra il «novello Asclepio» e il suo medium umano. 
epicureo $^{26}$, a cui il pamphlet è rivolto, e gli altri lettori colti di Luciano abbiano potuto accettare questa "mistificazione" e soprattutto se una tale invenzione fosse utile a raggiungere lo scopo, quello di combattere credulità e affannosa ricerca della parola oracolare che, in questo periodo, contagiavano non solo la massa degli incolti ma personaggi della stessa élite sociale e culturale cui Luciano e i suoi lettori appartenevano. Tali sono di fatto un Elio Aristide con il suo "cult-Verein" di retori e filosofi dell'Asclepieio di Pergamo ${ }^{27}$ e, qualche decennio più tardi, un Flavio Filostrato, con la sua costruzione -a beneficio del circolo filosofico dell'imperatrice Giulia Domna- dell'immagine dell"'uomo divino" Apollonio di Tiana, che a sua volta aveva avuto un ruolo non trascurabile nel panorama culturale e religioso del I sec. d.C. ${ }^{28}$. Sembra dunque che la studiosa, che spesso ironizza sulla "tentazione" di considerare storico il racconto lucianeo, confonda la satira con l'invenzione, senza valutare che la prima è possibile solo se si esercita su personaggi reali, e non solo su "tipi" generici, frutto di fantasia letteraria ${ }^{29}$. Mi sembra legittimo pertanto continuare a ritenere che dalla vivace descrizione lucianea -ricca di inventiva e di impietosa ironia- emerga l'immagine di una vera e propria "creazione religiosa" operata da un personaggio, Alessandro, che non pare del tutto digiuno proprio di quelle capacità intellettuali e di quel patrimonio culturale di cui le élites dei Sofisti andavano fiere. In essa convergono i tratti della più autorevole tradizione iatromantica e i vivaci tocchi innovativi che facevano dell'oracolo di Glicone un potente centro di richiamo non solo per le folle ansiose di risolvere i minuti e spesso angosciosi casi dell'esistenza quotidiana ma anche per autorevoli personaggi della vita politica, sociale e culturale del tempo, dalle cui decisioni dipendevano le sorti di comunità e di eserciti ovvero che ambivano a ricevere soluzioni a quesiti relativi agli stessi fondamenti dell'esistenza, nelle sue tensioni fra livello umano e divino. Pur riflessa nello specchio deformante della narrazione lucianea, l'immagine di Alessandro si impone all'attenzione dello storico come quella di una personalità religiosa

${ }^{26}$ Non mancano interpreti pronti a sospettare che si tratti di un"“invenzione" di Luciano e non di un reale personaggio. Anche in questa eventualità, peraltro, non si negherà che l'autore si rivolge a dei lettori in carne e ossa, capaci di valutare il senso delle sue affermazioni.

${ }^{27} \mathrm{La}$ documentazione discussa dalla studiosa, del resto, illustra la densa dimensione storicoculturale, oltre che religiosa, del fenomeno. Per quest'ultima cf. SFAMENI GASPARro (1998), oltre che i saggi editi da HARris-HOLMES (2008).

28 Oggetto di una letteratura critica assai ampia, la figura del saggio di Tiana e l'opera del suo "biografo" o meglio "agiografo" Filostrato rappresentano l'altra faccia della medaglia -encomiastica e celebrativa- raffigurante il tipo del theios aner tardo-antico, contrapposta a quella francamente denigratoria offerta da Luciano per l'altro "uomo divino" Alessandro. Osservazioni su questa opposizione in SFAMENI GASPARRO (2007) e (2012).

${ }^{29}$ Si può aggiungere ancora che, nell'eventualità di tale "invenzione", si dovrà spiegare la notizia lucianea della nozze fra Alessandro e la figlia del senatore romano P. Mummio Sisenna Rutiliano, personaggio storico senza dubbio, sopravvissuto al profeta e depositario della sua "eredità" religiosa (Alex., 61). Si può credere che l'autore, pur animato dall'intento satirico e abile creatore di «Racconti fantasticis, abbia "inventato" anche questo evento, potendo così essere facilmente smascherato dagli avversari contemporanei? 
creativa e originale, capace di confezionarsi con intelligente abilità e profonda conoscenza di autorevoli tradizioni mitico-cultuali una veste nuova, potentemente attrattiva perchè autentica cassa di risonanza di una serie vivace di istanze religiose contemporanee. Nell'ipotesi della mancanza di un preciso referente "storico" del testo lucianeo, anche se originalmente rivisitato alla luce di un'inventiva letteraria e retorica di singolare originalità, si dovrà concludere che lo stesso Luciano sia stato il "creatore" dell'oracolo di Glicone, per l'abile utilizzazione in esso di tutti gli elementi e temi tradizionali che un'analisi critica priva di pregiudizi e di precomprensioni è in grado di mettere in luce nella "confezione" di una identità religiosa quale è quella del «falso profeta Alessandro».

In particolare, il contenuto degli oracoli riferiti da Luciano, anche se fossero in tutto $\mathrm{o}$ in parte non autentici ${ }^{30}$, risulta comunque tale da riflettere lo stile e la tipologia di quelli che conosciamo promanare dai centri oracolari apollinei più accreditati. Paradossalmente si può dire che, se "falsi", gli oracoli in questione vedrebbero rafforzata la propria qualità di "testimoni" della diffusione e importanza di un genere oracolare dalle connotazioni teologiche nel panorama contemporaneo. Solo se tale "genere" avesse costituito una delle assi portanti del fenomeno divinatorio, infatti, un autore scaltrito come Luciano lo avrebbe imitato per costruire la sua immagine del "falso profeta". Quegli oracoli ci mostrano il dio di Abonutico e il suo profeta attenti a soddisfare- come l'Apollo delfico nella visione plutarchea- insieme le esigenze del bios e quelle del logos, mirando a risolvere sia i quotidiani problemi dell'esistenza del popolo sia gli interrogativi intellettuali degli uomini colti. Per tale via l'oracolo di Glicone si pone dunque in sintonia con il filone degli oracoli teologici che appunto in questo II sec. d.C. promana con varietà e ricchezza di espressioni dalle sedi di Claro e di Didima e trova voce più marcatamente speculativa nella raccolta degli Oracoli caldaici.

Una vena di religiosità neo-pitagorica emerge nella facies del profeta, già percepibile nel suo paragonarsi a Pitagora su cui ironizza Luciano ${ }^{31}$. A due personaggi, i quali si chiedevano se l'anima di Pitagora si fosse reincarnata nel

30 A parere di Victor (1997), in polemica con la conclusione di LANE Fox (1986, p. 234), i venticinque oracoli di Glicone addotti da Luciano non sarebbero frutto di un" "invenzione" ma piuttosto testimonianza dell'esistenza di una raccola oracolare scritta pertinente al santuario, come in tanti casi ben noti. Si può aggiungere che essi rientrano perfettamente nelle categorie individuate e analizzate da AUNE (1983, 19912, trad. it. 1996, p. 104-156), da quella degli «oracoli diagnostici» a quella dei responsi «di prescrizione», fino al «dialogo oracolare». Senza voler attribuire un carattere di "prova" dell'autenticità degli oracoli di Glicone a tale significativa serie di corrispondenze, non si può fare a meno di notare che l'inventiva di Luciano nel creare un così ampio numero di responsi secondo le più consolidate tipologie oracolari dovrebbe essere giudicata del tutto eccezionale.

31 Cf. Cumont (1922). In una lettera inviata al genero Rutiliano il personaggio avrebbe affermato «di essere simile a Pitagora» (Alex., 4) mentre nello svolgimento del complesso rituale misterico da lui istituito in evidente imitazione del modello eleusino, Alessandro danzava dinnanzi ai fedeli facendo «vedere la sua coscia d'oro, scoprendola ad arte» (Alex., 40). Si tratta del noto attributo del filosofo conferitogli dalla "leggenda" costruita attorno alla sua figura nel corso dei secoli. 
profeta, l'oracolo risponde: «L'anima di Pitagora ora si consuma ora riprende vigore. L'anima profetica, invece, è emanazione della mente divina. E l'inviò il padre in soccorso agli uomini buoni, e di nuovo a Zeus ritornerà colpita dal fulmine di Zeus» ${ }^{32}$ [App. A II, 5]. Sebbene nulla possa garantire o smentire l'autenticità di tale responso, è indubbio che chi lo ha formulato (Luciano, Alessandro?) era perfettamente consapevole di uno stile oracolare assai specifico, attento a problemi di pregnante densità teologica e antropologica. In questo linguaggio, che riconosce nell'identità profetica di Alessandro un'emanazione della stessa intelligenza divina, si colgono infatti peculiari assonanze con la letteratura oracolare "teologica" che aveva nell'Apollo clario il suo più autorevole titolare. Del resto, la nozione di una possibile "reincarnazione" in un personaggio attuale dalle straordinarie facoltà spirituali di uno dei grandi filosofi antichi, Pitagora appunto ovvero Platone, si integra perfettamente nell'orizzonte ideologico e religioso del II sec. d.C. Come è noto, il tema interviene nella complessa vicenda religiosa e letteraria dei due Giuliano, l'uno -il padre- detto «il Caldeo» e l'altro -il figlio- detto «il Teurgo», al quale la tradizione attribuisce la composizione degli Oracoli Caldaic ${ }^{33}$. Infatti il giovane Giuliano avrebbe ricevuto, nel corso di un rituale teurgico celebrato dal padre, un'anima arcangelica per l'intervento di Platone, appartenente alla medesima «catena spirituale» che risaliva ad Hermes ${ }^{34}$.

Una singolare analogia con la situazione di Alessandro descritta nell'oracolo citato mostra inoltre la condizione di un certo Ofellio Laetus, che un'iscrizione di Efeso definisce "filosofo platonico» ed esalta come colui nel quale rivive «se secondo Pitagora l'anima si trasforma (o: trasmigra) in un altro [corpo]»proprio l'anima di Platone ${ }^{35}$. Lo stesso personaggio è ricordato in un'iscrizione ateniese che lo definisce «teologo» e autore di un inno metarsios, ossia relativo alla realtà trascendente, al cui suono il cielo si aprì, rivelando agli uomini «la forma» (eidos) di tale realtà ${ }^{36}$.

Nello stile della letteratura oracolare teologica e in conformità alle istanze religiose che essa esprimeva risulta configurato un lungo colloquio tra Glicone e un personaggio di nome Sacerdote, la cui trascrizione il narratore/Luciano afferma di aver personalmente letto, incisa a lettere auree, nella casa del personaggio, nella città di Tio. Il consultante ha posto all'oracolo una domanda che si colloca in una tipologia ben nota da molti qualificati esempi, quali l'interrogativo sulla natura di Iao posto all'oracolo clario secondo la testimonianza

32 Alex., 40.

33 Souda, s.v. Ioulianos (ADLER II A [1928], 433-434, p. 641, 32-35). Nato sotto Traiano (98-117 d.C.), Giuliano il Teurgo giunse alla sua acmé al tempo di Marco Aurelio, quindi nel medesimo periodo di Alessandro. Ed. di DES PLACES (1971) e una messa a punto dei principali problemi interpretativi in SENG, TARDIEU (2010).

34 Psello, opusc. 46, 164-168, ed. J.M. Duffy, D.J. O’MEARA, Michaeli Pselli Philosophica minora I Opuscula logica, physica, allegorica, Stuttgart/Leipzig 1992. Cf. LANZI (2004/2005).

35 Cf. Nollé (1981).

36 BOWERSOCK (1984): entrambe le iscrizioni si datano nel I sec. d.C. 
di Macrobio $^{37}$, e quello avanzato nel III sec. d.C. dal soldato romano Massimo al dio solare egiziano Mandulis Aion. Nel significativo corpus delle iscrizioni rinvenute nella località di Talmis, in Nubia, nella sede oracolare del dio Mandulis, associato con Iside, soprattutto importanti per la loro connotazione "teologica", in senso fortemente cosmosofico, sono due testi. Il primo è il lungo poema composto dal soldato Massimo, che narra la propria esperienza di un sogno incubatorio, con apparizione di Mandulis che gli manifesta la propria natura solare $^{38}$. Il secondo documento è un racconto anonimo in prosa ritmica, forse opera dello stesso Massimo, che celebra il dio come «Signore che scocca i raggi (aktinobole), Mandoulis, Titano, Makareus» il cui autore dichiara di essersi recato al santuario «volendo conoscere con sicurezza se tu sei il Sole». Dopo essersi purificato, egli riceve in sogno la rivelazione della natura divina: «Allora ti ho conosciuto, Mandoulis, Sole, il sovrano che tutto vede, re dell'universo, l'Aion che domina su tutte le cose» ${ }^{39}$. L'esigenza di conoscere la vera identità del dio a cui ci si rivolge emerge anche dalle affermazioni (forse in un contesto di interrogazione oracolare a Bes o a Serapide) di un graffito del Memnonion di Abido (età romana): «Alcuni dicono che tu sei Asclepio. Ma io (credo) che tu sei Dioniso; altri che tu sei Phoibos ed Hermes e Arpocrate» ${ }^{40}$.

In questo sfondo religioso di ricerca della diretta manifestazione divina in un incontro "faccia a faccia" di cui la letteratura dei Papiri Greci Magici ci offre ampia testimonianza, si situa anche la complessa consultazione oracolare e relativa epifania divina di Asclepio ricercata con grande impegno alla fine premiato da parte del medico Tessalos (I sec. d.C. ${ }^{41}$.

Sacerdote interroga Glicone sulla sua identità [App. A I, 2]: «Dimmi dunque, o Glicone signore, chi sei?», esprimendo in tal modo quella fondamentale esigenza di conoscere natura e prerogative del proprio divino interlocutore da parte del fedele che a lui chiede l'instaurazione di un personale e intenso rapporto di familiarità per trovare risposta ai propri dubbi intellettuali ed esistenziali. Al dio che gli rivela di essere «il nuovo Asclepio» l'uomo propone ancora domande sulla veridicità attuale degli oracoli apollinei di Didima, Claro e Delfi e sulla propria

37 Sat. I, 18, $19-21$ ed. e trad. di MARINONE (19772), p. 272-273. Nel vasto e variegato quadro religioso, a forte impronta solare delineato nei Saturnalia, senza indicare la propria fonte, l'autore riferisce un «oracolo di Serapide» che recita: «Serapide, proclamato dagli Egiziani il massimo dio, quando Nicocreonte, re di Cipro, gli chiese quale dio si dovesse riconoscere in lui, soddisfece lo scrupolo religioso del re con questi versi: 'Quale dio io sia, apprendi da ciò che dico:/ la mia testa è la volta celeste, il mio ventre il mare,/ i miei piedi la terra e le mie orecchie stanno nell'aria/ e il mio sguardo lungi splendente è la luce brillante del sole’» (Sat. I, 20, 16-17, p. 284-285).

38 BERNAND (1969), $\mathrm{n}^{\circ} 168$. Tra gli studi su questo importante documento soprattutto significativi sono quelli di NOCK (1934) e DUNAND (2002).

${ }^{39}$ BERNAND (1969), n 166.

40 Rutherford (2003), p. 181.

${ }^{41}$ Festugière $(1967,1991)$, p. 143-169. Per una discussione dell'episodio e della relativa documentazione cf. MOYer (2003) e (2011); SFAMENI GASPARRo (2007a) e (2009). 
identità, secondo il già illustrato presupposto della teoria delle successive forme di vita cui l'anima è soggetta ${ }^{42}$. L'uso di redigere iscrizioni contenenti i responsi oracolari ricevuti è tanto diffuso da non richiedere particolari esemplificazioni, e come dimostra il caso di Enoanda ${ }^{43}$ - a partire dal II sec.d.C. esso si estende anche a quelli teologici. La notorietà del tema del destino dell'anima oltre la morte quale soggetto di interrogazione e risposta oracolare è confermata dal famoso oracolo di Apollo che Porfirio riferisce nella Vita di Plotino come pronunciato dal dio in risposta alla domanda di Amelio, intesa a conoscere «dove soggiomava l'anima di Plotino» ${ }^{44}$. Non devo ora entrare nel merito dell'interpretazione di questo notissimo documento, oggetto di una letteratura critica di notevole valore scientifico. Basti soltanto averlo evocato a ulteriore conferma di un clima ideologico e religioso in cui bagna la vicenda del protagonista del testo lucianeo, che si rivela dunque uomo del proprio tempo e non frutto della fantasia di un abile letterato. Né il tema è ignoto agli oracoli raccolti dall'autore della Theosophia. Egli riferisce la risposta di Apollo ad un fedele che gli chiedeva se l'anima sopravviva alla morte e ne ricevette una rassicurante risposta: dopo essersi liberata dai legami corporei, per decreto della divina Provvidenza, «essa è condotta nell'etere, immortale, e rimane per sempre protetta» da ogni dolore ${ }^{45}$.

Si può concludere che il testo di Luciano, nel farci conoscere un episodio importante della storia religiosa del mondo tardo-antico, anche se certo lascia più o meno ampi margini alla felice capacità inventiva dell'autore, delinea un quadro perfettamente coerente con lo scenario più ampio emergente dalle fonti contemporanee, di cui pertanto si dimostra buon testimone. Anche se questo quadro, infatti, non dovesse riflettere con assoluta fedeltà la "creazione" del profeta Alessandro certo è stato costruito dall'autore secondo quelli che possiamo riconoscere come i parametri generali di essa, ossia quale istituzione oracolare, ben organizzata con sede e personale sacro, da cui provenivano oracoli adatti a soddisfare gli interrogativi del logos oltre che dare sollievo ai molteplici e pressanti bisogni del bios.

Giulia SFAMENI GASPARRO

Università di Messina

Courriel:gasparro@unime.it

42 Alex., 43. A questa richiesta il dio risponde: «Sarai un cammello, poi un cavallo, poi un uomo sapiente e un profeta, non inferiore ad Alessandro» [App. A I, 2 e III, 3]. Una conferma dell'interesse del profeta per la tematica antropologica, in un quadro implicante successive incorporazioni dell'anima, è offerta da un oracolo dato a Rutiliano (Alex., 34: App. A III, 2).

${ }^{43}$ Livrea (1988); SuÁreZ de LA Torre (2010).

${ }^{44}$ Porfirio, Vita Plotini, 22. Cf. la traduzione di Brisson (2010), p. 303-307 con bibliografia.

45 Theos. BATIFFol (1916) $\mathrm{n}^{\circ}$ XIII = BeAtrice (2001), $\mathrm{n}^{\circ} 34$. L'oracolo apollineo è addotto da Lattanzio, Div. Inst. VII, 13, 15. 


\section{Appendice}

\section{Gli oracoli di Glicone}

\section{A. Oracoli teologici: le aporie del logos}

\section{Auto-proclamazione, nome e prerogative della divinità}

1. Alex., 18: Io sono Glicone, terzo sangue di Zeus, luce per gli uomini.

2. Alex., 43: (dialogo oracolare: Glicone e Sacerdote di Tio): Dimmi dunque, o Glicone signore, chi sei? - Io sono il nuovo Asclepio - Cosa intendi dire, un altro rispetto a quello venuto prima di te? - Non ti è certo lecito saperlo - Quanti anni rimarrai presso di noi a dare oracoli? - Tre oltre a mille - E poi dove ti trasferirai? - A Battra e nella regione circostante; bisogna che anche i barbari godano della mia presenza - E negli altri oracoli, a Didima, Claro e Delfi, è ancora tuo padre Apollo a pronunciare i vaticini, oppure sono falsi i responsi che si danno adesso in quei luoghi? - Non cercare di sapere neppure ciò: non è lecito - E io che forma avrò dopo questa vita? - Sarai un cammello, poi un cavallo, poi un uomo sapiente e un profeta, non inferiore ad Alessandro.

\section{Identità profetica}

1. Alex., 11: Perseide di stirpe, caro a Febo, ecco alla vostra presenza / il divino Alessandro, che per volere del fato ha il sangue di Podalirio.

2. Alex., 11: Oracolo della Sibilla: Sulle rive del Ponto Eussino, presso Sinope, / nascerà un profeta dov'è la Torre, sotto il regno degli Ausoni,/ mostrando dopo una prima unità e tre decine / altre cinque unità e una ventina triplicata, / nome a quattro elementi di un difensore.

3. Alex., 22: Tutto si compirà quando lo vorrò io e Alessandro, il mio profeta, me lo avrà chiesto e mi avrà supplicato intercedendo per voi.

4. Alex., 24: Ordino di onorare il mio servo e interprete; / perché non amo la ricchezza ma il mio profeta.

5. Alex., 40: L'anima di Pitagora ora si consuma ora riprende vigore. L'anima profetica, invece, è emanazione della mente divina. E l'inviò il padre in soccorso agli uomini buoni, e di nuovo a Zeus ritornerà colpita dal fulmine di Zeus.

\section{Natura e destino dell'anima}

1. Alex., 25: («Riguardo a Epicuro...quando un tale gli domandò quale fosse la vita di Epicuro nell'Ade, rispose:») Giace nel fango in ceppi di piombo.

2. Alex., 34: (a Rutiliano) Prima tu fosti il Pelide, poi Menandro, / poi colui che appari ora, e in seguito tu sarai raggio di sole / e vivrai ottant'anni oltre i cento.

3. Alex., 43: (dialogo oracolare: Glicone e Sacerdote di Tio) ... E io che forma avrò dopo questa vita? - Sarai un cammello, poi un cavallo, poi un uomo sapiente e un profeta, non inferiore ad Alessandro.

\section{L'oracolo fonte di verità?}

1. Alex., 29: Ora vai a Claro, ad ascoltare la voce di mio padre.

2. Alex., 29: Accostati ai penetrali dei Branchidi e ascolta i responsi.

3. Alex., 29: Recati a Mallo e dagli oracoli di Anfiloco. 
4. Alex., 42: (dialogo oracolare: Glicone e Sacerdote di Tio) ... E negli altri oracoli, a Didima, Claro e Delfi, è ancora tuo padre Apollo a pronunciare i vaticini, oppure sono falsi i responsi che si danno adesso in quei luoghi?- Non cercare di sapere neppure ciò: non è lecito.

\section{$V$. Sulla retta dottrina: condanna delle empie dottrine di Epicuro}

1. Alex., 47: Ordino di distruggere col fuoco le massime del vecchio che non vede.

\section{B. Oracoli per il bios}

\section{Iatromantica}

1. Alex., 25: Spargi di comino la malva in una sacra pentola di maialini.

2. Alex., 28: Non cercare più rimedio al tuo male funesto, / perché il destino di morte è evidente e non puoi sfuggire.

3. Alex., 52: Una forma eubargoulis nell'ombra chnechikraghe lascerà la tua terra.

4. Alex., 53: sabardalachou malachaattealos era.

5. Alex., 53: Ti raccomando di spalmarti con cimitide e saliva di destriero.

II. Oracoli profilattici

1. Alex. 36: (per la peste del 166 d.C.); Febo dalle chiome intonse tiene lontano la nube della pestilenza.

\section{Oracoli per le incertezze e crisi dell'individuo e della comunità}

a. Le grandi imprese militari

1. Alex., 27: (per il senatore Marco Sedazio Severiano: sull'esito della campagna militare contro i Parti, a. 161 d.C.) Sottomessi Parti e Armeni con rapida lancia / tornerai a Roma e al limpido Tevere / cinto le tempie di una corona di raggi.

2. Alex., 27: (oracolo post-eventum, sostitutivo del precedente) Tu non lanciare l'esercito contro gli Armeni, è meglio non farlo, / perché un uomo vestito con tunica muliebre, scoccando dall'arco / destino di morte, non ti tolga la vita e la luce.

3. Alex., 48: (per la guerra di Marco Aurelio contro Quadi e Marcomanni) Nei gorghi dell'Istro, fiume che nasce da Zeus, / ordino di gettare due ministri di Cibele, / fiere che vivono sui monti, e quanti fiori ed erbe odorose/ fa crescere l'aria dell'India; subito saranno vittoria e gloria grande con serena pace.

b. I problemi degli individui

4. Alex., 33: (quale maestro per un giovane?) Pitagora e il nobile aedo che canta le guerre.

5. Alex., 35: («a Rutiliano che gli chiedeva se dovesse sposarsi») Sposa la figlia di Alessandro e di Selene.

6. Alex., 43: (a Sacerdote di Tio) Non prestare fede a Lepido, poiché destino luttuoso l'accompagna.

7. Alex., 50: Vuoi sapere chi in gran segreto a casa tua,/ sul tuo letto, fa l'amore con tua moglie Calligeneia?/ E' lo schiavo Protogene, nel quale hai fiducia. / Tu infatti ti sei goduto lui ed egli a sua volta/ gode tua moglie, ricambiando l'offesa subita. / Hanno già preparato un veleno mortale/ perché tu non senta né veda ciò che fanno: / lo troverai sotto il letto, vicino al muro/ dove sta la testa; e la serva Calipso è complice.

8. Alex., 53: Tu non andare per mare ma viaggia per via di terra.

9. Alex., 54: Gode di vagabondi amori e di letti impuri. 


\section{Bibliografia}

W. Ameling (1985), «Ein Altar für Alexander von Abonuteichos», EA 5, p. 34-36.

D.E. Aune (1983, 1991 ${ }^{2}$, 1996), Prophecy in Early Christianity and the Ancient Mediterranean World, Grand Rapids; trad. it., Brescia.

E. BABELON (1900), «Le faux prophète Alexandre d'Abonotichos», RN s. IV, 4, p. 1-30.

P. BATIFFol (1916), «Oracula hellenica», RBi 25, p. 177-199.

P.F. BeAtrice (2001), Anonymi Monophysitae Theosopbia. An Attempt to Reconstruction, Leiden.

A. Benduin (2006, 2011), «Von Nutzen und Nachteil der Mantik. Orakel im Medium von Handlung und Literatur in der Zeit der Zweiten Sophistik», in D. ELM VON DER OsTEN, J. RÜPKE, K. WALDNER (ed.), Texte als Medium und Reflexion von Religion im römischen Reich, Stuttgart (PAwB, 14), p. 159-207; rist. «On the Use and Disadvantages of Divination. Oracles and their Literary Representations in the Time of the Second Sophistic», in J.N. NORTH, S.R.F. PrICE (ed.), Oxford Readings in Classical Studies. The Religious History of the Roman Empire. Pagans, Jews, and Christians, Oxford, p. 175-250.

É. BERNAND (1969), Inscriptions métriques de l'Égypte gréco-romaine. Recherches sur la poésie épigrammatique des Grecs en Égypte, Paris.

J. BOMPAIRE (1958), Lucien écrivain. Imitation et création, Paris.

G. Bordenache BatTaglia (1988), s.v. «Glykon», LIMC, IV, 1, p. 278-283; IV, 2, p. 161-162.

G.W. Bowersock (1982), «Plutarch and the Sublime Hymn of Ofellius Laetus», GRBS 23, p. $275-279$.

B. Branham (1989), Unruly Eloquence: Lucian and the Comedy of Tradition, Cambridge MA.

L. Brisson (2010), «Porphyre Sur la vie de Plotin et la mise en ordre de ses livres. Présentation, traduction et notes», in L. Brisson, J.-Fr. Pradeau (dir.), Plotin. Traités 51-54 Porphyre, Vie de Plotin, Paris, p. 257-335.

K. BURESCH (1889), Klaros. Untersuchungen zum Orakelwesen des späteren Altertums, Leipzig; rist. Aalen 1973.

A. Busine (2005), Paroles d'Apollon. Pratiques et traditions oraculaires dans l'Antiquité tardive $\left(I I^{e}-V T^{e}\right.$ siècles), Leiden-Boston (RGRW, 156).

M. CASTER (1937), Lucien et la pensée religieuse de son temps, Paris.

- (1938), Études sur Alexandre ou le faux prophète de Lucien, Paris.

M. Caster, P.-E. DauZat (2001, 2002²), Lucien. Alexandre ou le faux prophète, Paris.

A. Chaniotis (2002), «Old Wine in a New Skin: Tradition and Innovation in the Cult Foundation of Alexander of Abonouteichos", in E. DABROWA (ed.), Tradition and Innovation in the Ancient World, Cracovia (Electrum, 6), p. 67-85.

F. Cumont (1887), Alexandre d'Abonotichos. Un épisode de l'bistoire du paganisme au Ile siècle de notre ère, Mémoires couronnés et autres Mémoires publiés par l'Académie royale de Belgique, p. 3-54.

— (1922), «Alexandre d'Abonotichos et le néo-pythagorisme», RHR 86, p. 202-210.

D. DEl Corno, L. CAmpolunghi (1992), Luciano di Samosata Alessandro o Il falso profeta, Milano.

É. DES PlaCes (1971), Oracles chaldä̈ques, Paris.

F. Dunand (2002), «Le désir de connaitre Dieu. Une vision de Mandoulis au temple de Kalabscha», in F. Dunand, F. Boespflug (ed.), Voir les dieux Voir Dieu, Strasbourg, p. 23-38.

D. ELM VON DER OSTEN (2006), «Die Inszenierung des Betruges und seiner Entlarvung: Divination und ihre Kritiker in Lukians Schrift <Alexandros oder der Lügenprophet $»$ «, in D. Elm von der Osten, J. Rüpke, K. Waldner (ed.), Texte als Medium und Reflexion von Religion im römischen Reich, Stuttgart (PAwB, 14), p. 141-157.

A.-J. Festugière (1967), «L'expérience religieuse du médecin Thessalos», in ID., Hermétisme et mystique païenne, Paris; tr. it. Genova 1991, p. 143-169.

R. FlaCelière (1974), Plutarque. CEuvres morales, T. VI Dialogues Pythiques, Paris. 
W.V. Harris, B. Holmes (2008) (ed.), Aelius Aristides between Greece, Rome, and the Gods, Leiden.

C. Howgego, V. Heuchert, A. Burnett (2005) (ed.), Coinage and Identity in the Roman Provinces, Oxford.

C.P. Jones (1986), Culture and Society in Lucian, Cambridge Mass./London.

S.I. Johnston (2008), Ancient Greek Divination, Oxford.

R. LANE Fox (1986, 1991), Pagans and Christians, London; trad. it. Roma/Bari.

S. LANZI (2004/2005), «La questione dei Giuliani e gli Oracoli caldaici: alcuni problemi storicoreligiosi», Mythos 12, p. 145-169.

E. LIVREA (1998), «Sull’iscrizione teosofica di Enoanda», ZPE 122, p. 90-96.

N. MARINONE $\left(1967,1977^{2}\right)$ (cur.), I Saturnali di Macrobio Teodosio, Torino.

A. Mastrocinque (1999), «Alessandro di Abonoutheichos e la magia», in N. Blanc, A. Buisson (ed.), Imago Antiquitatis. Religions et iconographie du monde romain. Mélanges offerts à Robert Turcan, Paris, p. 341-352.

- (2009), «Alessandro di Abonouteichos e il culto di Asclepio», in E. De Miro, G. Sfameni Gasparro, V. Calì (ed.), Il culto di Asclepio nell'area mediterranea, Atti del Convegno Internazionale Agrigento 20-22 novembre 2005, Roma 2010, p. 195-200.

A.V.B. Miron (1996), «Alexander von Abonuteichos. Zur Geschichte des Orakels des Neos Asklepios Glykon», in W. LeschHorn, A.V.B. Miron, A. Miron (ed.), Hellas und die griechische Osten. Studien zur Geschichte und Numismatike der griechischen Welt, Festschrift für P.R. Franke zum 90. Geburtstag, Sarrebruck, p. 153-188.

M. Monaca (2008) (cur.), Oracoli sibillini, Introduzione, traduzione e note, Roma (Testi patristici, 199).

C. Moreschini (1997) (cur.), Plutarco. L'E di Delfi, Napoli (Corpus Plutarchi Moralium, 27).

I. Moyer (2003), «Thessalos of Tralles and Cultural Exchange», in S. NoEgel, J. Walker, B. Wheeler (ed.), Prayer, Magic, and the Stars in the Ancient and Late Antique World, Pennsylvania, p. 39-56.

- (2008), «Thessalos and the Magic of Empire», in ID., Egypt and the Limits of Hellenism, Cambridge, p. 208-273.

J.M. a NieTo IbÁÑEZ (2010). Cristianismo y profecías de Apolo. Los oráculos paganos en la patristica griega (siglos II-V), Madrid.

A.D. NoCK (1928), «Oracles théologiques «, REA 30, p. 280-290; rist. in ID., 1972, p.160-168.

- (1928a), «Alexander of Abonuteichos», CQ 22, p. 160-162.

— (1934), «A Vision of Mandulis Aion», HTR 27, p. 53-104; rist. in ID., 1972, p. 357-400.

- (1972), Essays on Religion and the Ancient World. Selected and edited, with an Introduction, Bibliography of Nock's writings, and Indexes, by Z. Stewart, 2 vol., Oxford.

J. Nollé (1981), «Ofellius Laetus, platonische Philosoph», ZPE 41, p. 197-206.

A. Petsalis-Diomidis (2010), Truly beyond Wonders. Aelius Aristides and the Cult of Asclepius, Oxford.

S. PozzI (2003), «Sull'attendibilità del narratore nell'Alexander di Luciano», Prometheus 29, p. 129$150 ; 241-258$.

L. Robert (1980), «Lucien et son temps», in ID., A travers l'Asie Mineure. Poètes et prosateurs, monnaies grecques, voyageurs et géographie, Paris, p. 393-436.

— (1981), «Le serpent Glycon d'Abônouteichos à Athènes et Artémis d'Éphèse à Rome», CRAI, p. 513-535.

P. Robiano (2001), «Lucien, un témoignage-clé sur Apollonios de Tyane», RPh s. III, 77, p. 259273.

A. Robu (2007), «Réflexions sur le culte d'Apollon à Chalcédoine», LEC 75 [P. BonneChere, G. Cursaru (ed.), Actes de la VTe conférence annuelle de l'EASR et de la conférence spécial conjointe de l'LAHR, Bucarest, 20-23 september 2006], p. 137-155. 
I. Rutherford (2003), Pilgrimage in Greco-Roman Egypt: New Perspectives of Graffiti from the Mnemonion at Abidos, in R. MATTHews, C. Roemer (ed.), Ancient Perspectives on Egypt, London, p. 171-189.

J. SCHWARTz (1965), Biographie de Lucien de Samosate, Bruxelles (Coll. Latomus, 83).

H. Seng, M. TARDieu (2010) (ed.), Die Chaldaeischen Orakel: Kontext, Interpretation, Rezeption, Heidelberg.

G. SFAmeni Gasparro (1994), “"Religione” e "teologia": due "quantità" della ricerca storicoreligiosa», in U. BIANCHI (ed.), The Notion of «Religion» in Comparative Research. Selected Proceedings of the XVI IAHR Congress, Roma, p. 733-740.

- (1995), «Ispirazione delle Scritture e divinazione pagana: aspetti della polemica fra Origene e Celso», in G. Dorival, A. Le Boulluec (ed.), Origeniana Sexta. Origène et la Bible/Origen and the Bible, Leuven, p. 287-302; rist. in EAD., Origene e la tradizione origeniana in Occidente: letture storico-religiose, Roma, 1998 (Biblioteca di Scienze religiose, 142), p. 177-194.

- (1996), «Plutarco e la religione delfica: il dio "filosofo" e il suo esegeta», in I. GaLLo (ed.), Plutarco e la religione, Ravello, 29-31 maggio 1995, Napoli, p. 157-188; rist. con modifiche in EAD. (2002), p. 113-148.

- (1996 [1998]), «Alessandro di Abonutico lo "pseudo-profeta" ovvero come crearsi un'identità religiosa. I. Il profeta "eroe" e "uomo divino" ", SMSR 62 (= n.s. 20, 1/2), Omaggio a Dario Sabbatucci, Roma, 1998, p. 565-590; rist. con modifiche in EAD. (2002), p. 149-202.

- (1998), «Elio Aristide e Asclepio, un retore ed il suo dio: salute del corpo e direzione spirituale», in E. Dal Covolo, L. GiAnnetTo (cur.), Cultura e promozione umana. La cura del corpo e dello spirito nell'antichità classica e nei primi secoli cristiani. Un magistero ancora attuale?, Troina, p. 123-143; rist. con modifiche in EAD. (2002), p. 203-253.

- (1998a), «La Sibilla voce del dio per pagani, ebrei e cristiani: un modulo profetico al crocevia delle fedi», in I. Chirassi Colombo, T. Seppilli (ed.), Sibille e linguaggi oracolari. Mito Storia Tradizione, Pisa/Roma, p. 505-553; rist. con modifiche in EAD. (2002), p. 61-112.

- (1999), «Alessandro di Abonutico lo "pseudo-profeta" ovvero come costruirsi un'identità religiosa. II. L'oracolo e i misteri, in Les syncrétismes religieux dans le monde méditerranéen antique, Bruxelles-Rome, p. 275-305; rist. con modifiche in EAD. (2002), p. 149-202.

- (2002), Oracoli Profeti Sibille. Rivelazione e salvezza nel mondo antico, Roma (Biblioteca di Scienze religiose, 171).

- (2007), «Il sofista e l"uomo divino': Filostrato e la costruzione della «vera storia» di Apollonio di Tiana», in O.D. CordovanA, M. GALli (cur.), Arte e memoria culturale nella seconda Sofistica, Catania, p. 271-288.

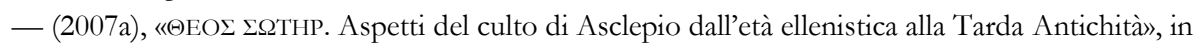
H. Brandenburg, St. Heid, Chr. Markschjes (ed.), Salute e guarigione nella Tarda Antichità, Città del Vaticano, p. 245-271.

- (2009), «Asclepio divinità epifanica e salvatrice: l'esperienza di Thessalos fra medicina, religione e magia», in E. De Miro, G. SFAmeni Gasparro, V. Calì (ed.), Il culto di Asclepio nell'area mediterranea, Roma, p. 287-311.

- (2010), Dio unico, pluralità e monarchia divina. Esperienze religiose e teologie nel mondo tardo-antico, Brescia.

- (2012), «Mediatori della conoscenza e operatori del Sacro nel mondo mediterraneo antico: "uomini divini", profeti, taumaturghi e maghi», in M. RIZZUTO, P. URIZZI (cur.), Rivelazione e conoscenza. Prospettive sacre d'Oriente e d'Occidente, Atti del $2^{\circ}$ Seminario di studi, Siracusa 6-9 dicembre 2006, Palermo, p. 21-70.

E. SuÁrez de la Torre (2002²), Oraculos Sibilinos, trad., intr. y notas, in A. Diez MaChO †, A. Piñero SÁenz (ed.), Apòcrifos del Antiguo Testamento, Madrid, vol. III, p. 329-612.

- (2010), «Tradition oraculaire et réflexion 'théosophique' dans l'oracle gravé à Oinoanda», in Ch. GuitTard (ed.), Le Monothéisme. Diversité, exclusivisme ou dialogue?, Paris, p. 107-129.

P. VAn Nuffelen (2011), Rethinking the Gods. Philosophical Readings of Religion in the Post-Hellenistic Period, Cambridge. 
U. VICTOR (1997), Lukian von Samosata. Alexandros oder der Lügenprophet, Leiden (RGRW, 132).

O. WeINREICH (1921), «Alexandros der Lügenprophet und seine Stellung in der Religiosität des II. Jahrhunderts n. Chr.», Neue Jahrbücher fur das klassische Altertums Geschicbte und Deutsche Literatur 47, p. 129-151; rist. in ID., Ausgewählte Schriften I 1907-1921, Amsterdam, 1969, p. 520551. 